\title{
The All Sky Automated Survey (ASAS-3) System - Its Operation and Preliminary Data
}

\author{
G. Pojmański \\ Warsaw University Astronomical Observatory, Al Ujazdowskie 4, \\ 00-478 Warszawa, Poland
}

\begin{abstract}
ASAS-3 is the next step in the All Sky Automated Survey. This paper describes the new hardware, consisting of two wide field $(9 \times 9$ deg) telephoto instruments and one $F=750 \mathrm{~mm} D=250 \mathrm{~mm}$ telescope with $2 \times 2 \mathrm{deg}$ FOV, each equipped with a $2 \mathrm{~K} \times 2 \mathrm{~K}$ CCD camera. Wide field instruments are now observing the whole southern sky (almost 30,000 sq. deg) at the average rate of 0.5 measurements per day. A narrow field instrument is connected to the GCN network and is ready to respond to the GRB alerts in tens of seconds. Preliminary photometric data are presented here and briefly discussed.
\end{abstract}

\section{Introduction}

The All Sky Automated Survey (Pojmański 1997, 1998, 2000) is an observing project which ultimate goal is the photometric monitoring of various objects all over the sky (Paczyński 1997, 2001). In the years from 1997-2000 a small, automated prototype instrument, equipped with a $768 \times 512$ MEADE Pictor 416 CCD camera, a $135 \mathrm{~mm}$ f/ 1.8 telephoto lens and an $I$-band (Schott RG-9, $3 \mathrm{~mm}$ ) filter has been monitored $0.7 \%$ of the sky, to the limiting magnitude of $I \sim 13$. During 3 years of operation it has collected over $50 \times 10^{6}$ measurements of over 150,000 stars, detecting almost 4000 variable stars.

This prototype instrument, its data acquisition and reduction pipeline and the ASAS Catalog have been described by Pojmański (1997). Some first results of the search for short time scale periodic variables were presented by Pojmański (1998) while results of the search for long term variables in the ASAS Selected Fields, using data obtained during the first two years of the prototype instrument's operation, were summarized by Pojmański (2000).

After completing the prototype phase of the project in early 2000 we installed an upgraded ASAS-3 system in the dome of the 10" astrograph in the Las Campanas Observatory (operated by the Carnegie Institution of Washington). The new hardware consists of three independent instruments (Fig. 1), each equipped with an automated parallactic mount, imaging optics with a standard filter, a $2 \mathrm{~K} \times 2 \mathrm{~K}$ CCD camera (AP-10 from Apogee) and a dedicated computer (Dual Pentium-3 $650 \mathrm{MHz}$ Linux box, $128 \mathrm{RAM}, 35 \mathrm{~GB}$ hard disk). There is one DAT-3 tape storage for all three systems, and a simple surveillance camera connected to the Internet. 


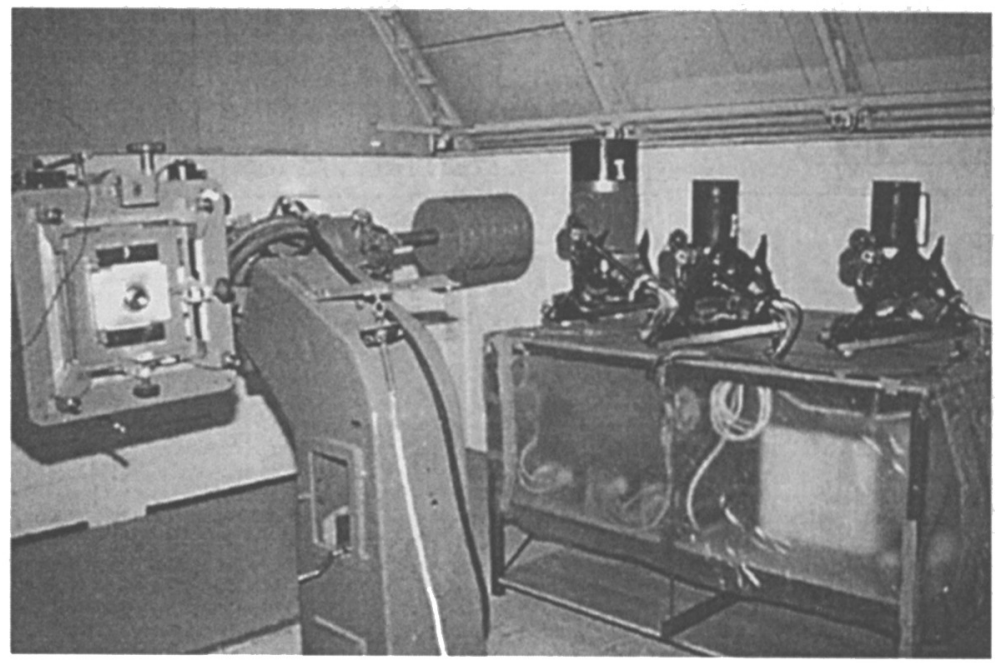

Figure 1. ASAS-3 in the 10" astrograph dome. From the left: 10" astrograph, $F=750 \mathrm{~mm}$ telescope, 2 wide-field instruments with $200 / 2.8$ telephoto lenses.

\section{Instrumentation}

\subsection{Mount}

The equatorial mount has a compact ( $500 \mathrm{~mm}$ side) horseshoe design driven in both axes by stepper motors, intermediary sprocket gears and friction rollers. It is controlled by a dedicated electronic box containing power supplies, microprocessor controller and translator drives. Both stepper motors are controlled over the same serial link.

The RA drive transmission rate was designed for 1.5 arcsec steps, so it requires about 10 steps per second to track the sky rotation. The sprocket gears and friction roller form a non-backlash drive with a very good positioning repeatability. The declination drive has a much smaller gear rate, so that each step corresponds to about 12.5 arcsec on the sky.

The maximum slewing rate is about $5 \mathrm{deg} / \mathrm{sec}$ in hour angle and $40 \mathrm{deg} / \mathrm{sec}$ in declination. The average pointing time during a regular observing run is about 10 seconds.

The instrument bay is $280 \mathrm{~mm}$ in diameter and the total weight of the imaging equipment is less then $15 \mathrm{~kg}$.

\subsection{CCD Camera}

Three $2 \mathrm{~K} \times 2 \mathrm{~K}$ (14 um) commercial cameras were purchased for the project. High read-out noise $\left(>10 e^{-}\right)$and 14 bit $\mathrm{ADC}$ were accepted as a trade-off for a 5 sec read-out time. Each camera is thermoelectrically cooled to $-20 \mathrm{C}$ during night operation. Unfortunately two of the three systems do not yet meet factory 


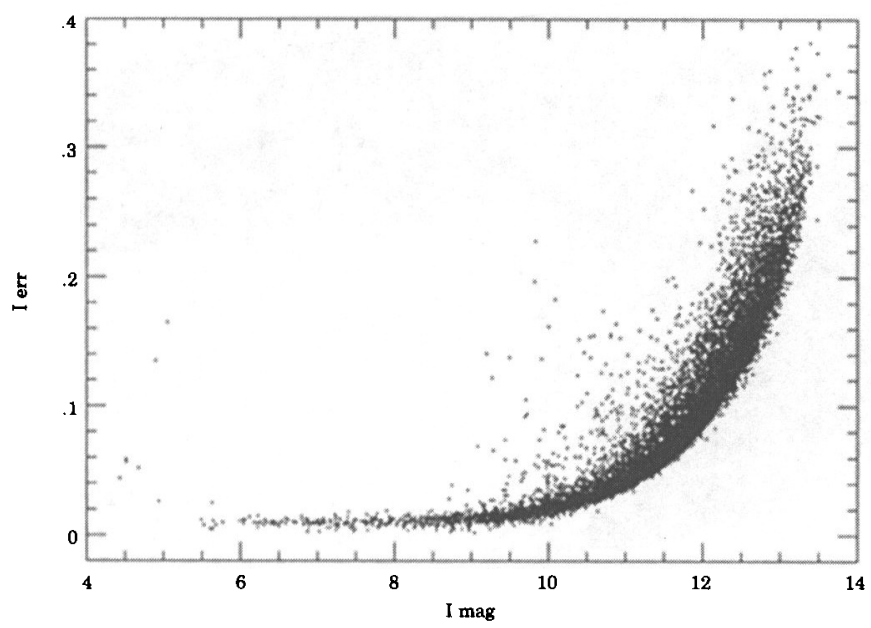

Figure 2. Standard deviation $\sigma_{I}$ of the stellar magnitudes $v s I$-band magnitudes for LMC stars observed with the wide-field $I$ camera and 1-minute exposures.

specifications and show twice as much read-out noise as desired, hence reducing system sensitivity. Each camera system is linked to a dedicated Dual Pentium-3 $650 \mathrm{MHz}$ Linux box, which handles both telescope and camera control, data acquisition and data reduction.

\subsection{Imaging Optics}

The two wide-field systems are equipped with the Minolta 200/2.8 APO-G telephoto lenses. These provide superb sharpness (in fact better than needed: the $F W H M$ of stellar images is only 1.3-1.8 pixels) but unfortunately also strong vignetting ( $40-50 \%$ in the corners). With the $2 \mathrm{~K} \times 2 \mathrm{~K}$ CCD, each camera captures $8.8 \times 8.8 \mathrm{deg}$ of the sky. One system is equipped with a standard $I$ filter and the other with $V$.

The narrow field instrument is a $D=250 \mathrm{~mm}, F=750 \mathrm{~mm}$ Cassegrain system with a three element, Wyne-type field corrector. It gives sharp images $(F W H M<2.2$ pixels) within a 2 deg diameter field. With the $2 \mathrm{~K} \times 2 \mathrm{~K} \mathrm{CCD}$, the field of view is $2.2 \times 2.2 \mathrm{deg}$. This system has an $I$ filter in its optical path.

\section{Performance}

For 1 minute exposures the limiting magnitudes for the wide-field $V$, wide-field $I$ and Cassegrain $I$ systems are 13.5, 13.2 and 14.8, respectively (Fig. 2). The last two numbers should improve by $0.5-1$ magnitudes once problems with the high read-out noise are fixed.

The mechanical quality of the mount allows for exposures as long as 5-10 minutes for wide-field systems and about 3 minutes for the Cassegrain telescope, 


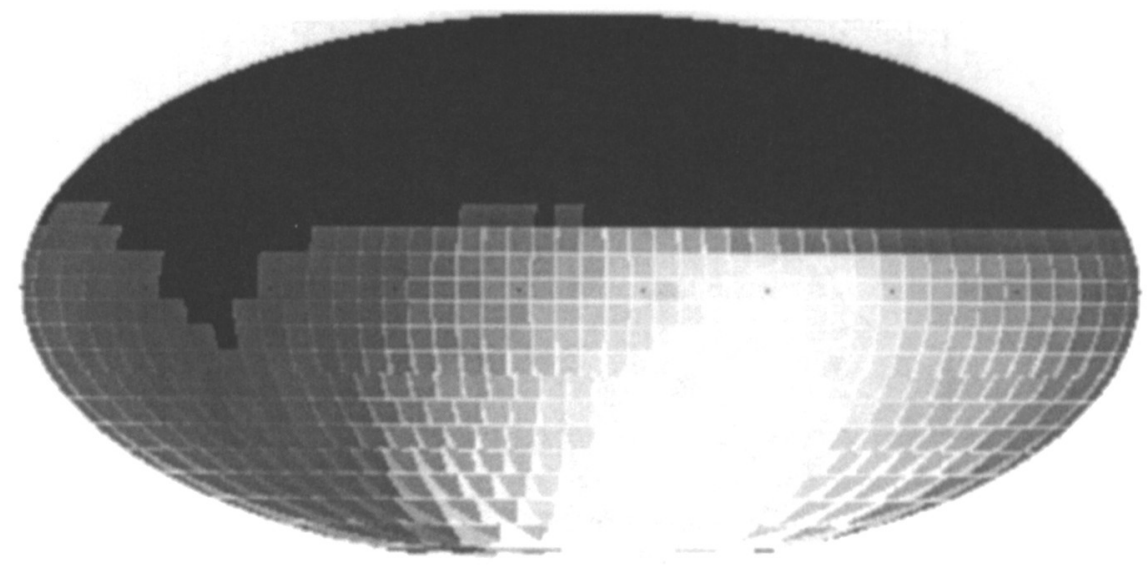

Figure 3. Coverage of the sky after three months of ASAS-3 operation. The white color corresponds to areas observed at least 70 times.

without any substantial degradation in the PSF of the stellar images. The relatively large Cassegrain system is sensitive to moderate winds $(>7 \mathrm{~m} / \mathrm{s})$, in which case slightly elongated images are produced.

The pointing accuracy of the mounts (without a pointing model being applied) is a few arc minutes in both axes.

\section{Observations}

The whole sky has been divided into $7098 \times 8 \mathrm{deg}$ fields, of which $500(70 \%)$ can be observed from Las Campanas in the $I$-band and about $420(60 \%)$ in the $V$-band (the difference is due to dome obscuration). During a single summer night one can observe up to 300 different fields.

Weather indications to start/stop observations are obtained automatically from the Polish OGLE telescope project (Udalski et al. 1997). If that telescope is not operating, we use the nearby La Silla (ESO) WWW weather service instead.

A routine observing schedule for each wide-field camera consists of cycling through the list of fields, and picking the one that is the most suitable for observation at the moment.

The default exposure time for the $I$ systems is 2 minutes, while for the wide-field $V$ system it is 3 minutes. Taking into account the overhead read-out and pointing time we can take between 160 and 200 frames per night with the $V$ system and between 230 and 300 frames per night with the $I$ systems (depending on the season). This means that we can cover the whole available sky with both filters in one or two days.

After the initial whole-sky observations, we will change the above schedule slightly to search for short-period variability in each field. 

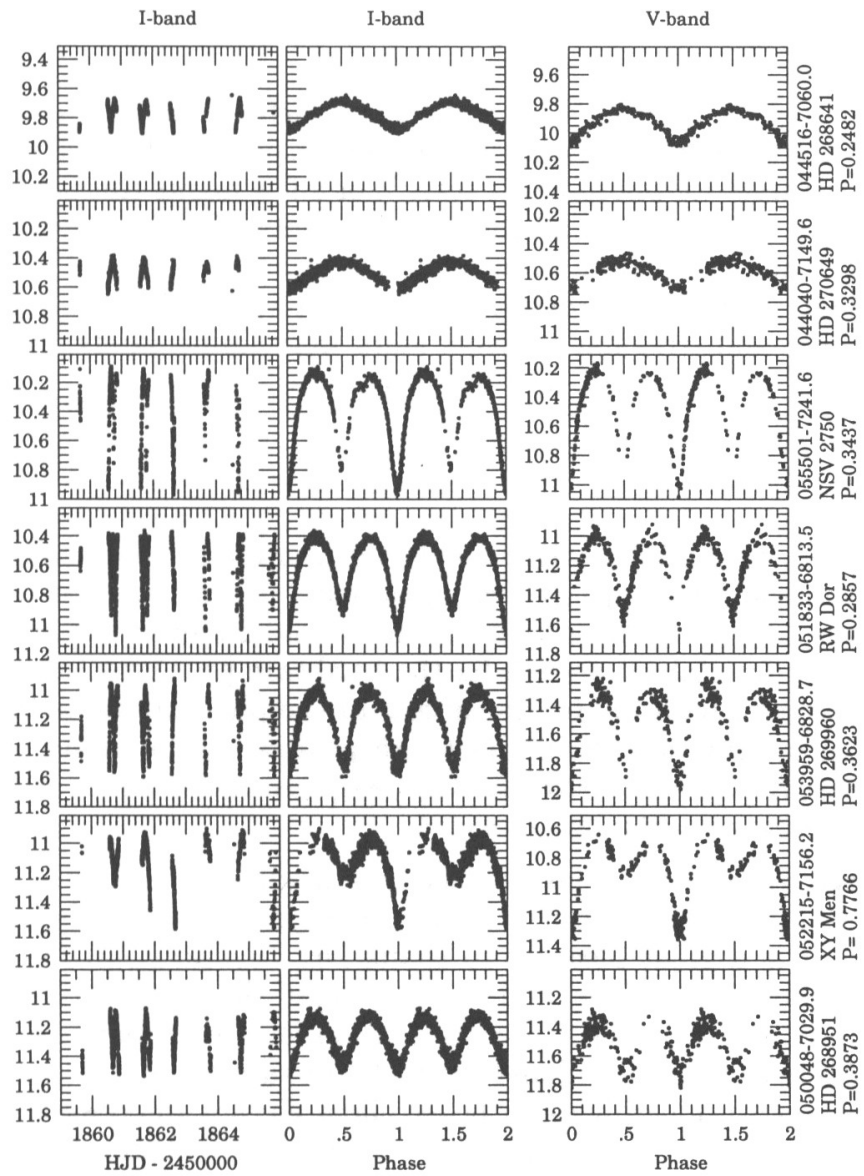

Figure 4. Periodic variable stars observed by ASAS-3 instruments in the LMC and SMC fields. The ASAS name, cross-identification and periods are printed on the right side of the panel.

The narrow-field instrument is connected directly to the GRB Coordinates Network (Barthelmy et al. 1998). Follow-up observations will start about ten seconds after receiving a GRB alert. Between alerts a set of preselected fields of interest is observed.

The ASAS-3 system currently takes about 600 frames per night, which means a raw data stream of 5 GB per night. The loss-less compression reduces this stream to 2 GB per night (more than one DAT-3 tape per week). We are therefore considering a compression tolerating loss in future.

Up to February 2001, after the first months of unattended operation, over 400 fields were observed. Some 15000 frames from each of the wide-field $I$ and Cassegrain $I$ systems and 11000 frames from the wide-field $V$ system were collected, covering $95 \%$ of the observable sky, on an average of nearly 40 times (Fig. 3). 


\section{First Variables}

Before the regular observing schedule has started, we have collected several thousand images of various fields for testing purposes. The LMC field was used as a primary target for photometric tests. For a few nights this field was observed with 1 minute exposures by all three instruments. The data were reduced and analyzed by the default data processing pipe-line.

Although only a very provisional search for variability has been performed, a few periodic and irregular variable stars were immediately identified in the data (Fig. 4) - some of them new bright binaries.

\section{Prospects}

In a few weeks the ASAS-3 will complete the preliminary data collection process for the entire southern sky. At this point some manual work will be necessary to create the best template catalog of the $\mathrm{V}$ and I measurements. We plan to stop observing for a short period, fix the hardware problems (noise) and finally restart the system with full on-line access to the photometric database.

Current information about the ASAS project can be found on the WWW home page at http://www.astrouw.edu.pl/ gp/asas/asas.html or http://archive.princeton.edu/ asas/

Acknowledgments. This project was made possible by a generous gift from Mr. William Golden to Dr. Bohdan Paczyński, and funds from Princeton University. It is a great pleasure to thank Dr. B. Paczyński for his initiative, interest, valuable discussions, and the funding of this project.

I am indebted to the OGLE collaboration for the support and maintenance of the ASAS instrumentation, and to the Observatories of the Carnegie Institution of Washington for providing the excellent site for the observations.

This work was partly supported by the KBN 2P03D01416 grant.

\section{References}

Barthelmy, S.D., et al. 1998, in Gamma-Ray Bursts: 4th Huntsville Symposium; ed. Charles A. Meegan, Robert D. Preece, and Thomas M. Koshut, 99

Paczyński, B. 1997, in Proceedings of 12th IAP Colloquium, Variable Stars and the Astrophysical Returns of Microlensing Searches, ed. R. Ferlet, 357

Paczyński, B. 2001, these proceedings

Pojmański, G. 1997, Acta Astr., 47, 467

Pojmański, G. 1998, Acta Astr., 48, 35

Pojmański, G. 2000, Acta Astr., 50, 177

Udalski, A. Kubiak, M., and Szymański, M. 1997, Acta Astr.,47, 319 